$11-1-2016$

\title{
Improved Ridge Estimator in Linear Regression with Multicollinearity, Heteroscedastic Errors and Outliers
}

\author{
Ashok Vithoba Dorugade \\ Y C Mahavidyalaya, Halkarni, Tal-Chandgad, Kolhapur, Maharashtra, India, adorugade@rediffmail.com
}

Follow this and additional works at: http://digitalcommons.wayne.edu/jmasm

Part of the Applied Statistics Commons, Social and Behavioral Sciences Commons, and the Statistical Theory Commons

\section{Recommended Citation}

Dorugade, Ashok Vithoba (2016) "Improved Ridge Estimator in Linear Regression with Multicollinearity, Heteroscedastic Errors and Outliers," Journal of Modern Applied Statistical Methods: Vol. 15 : Iss. 2 , Article 23.

DOI: $10.22237 /$ jmasm/1478002860

Available at: http://digitalcommons.wayne.edu/jmasm/vol15/iss2/23

This Regular Article is brought to you for free and open access by the Open Access Journals at DigitalCommons@WayneState. It has been accepted for inclusion in Journal of Modern Applied Statistical Methods by an authorized editor of DigitalCommons@WayneState. 


\section{Improved Ridge Estimator in Linear Regression with Multicollinearity, Heteroscedastic Errors and Outliers}

\section{Cover Page Footnote}

A.V. Dorugade Department of Statistics, Y C Mahavidyalaya Halkarni, Tal-Chandgad, Dist-Kolhapur, Maharashtra, India -416552. Email: adorugade@rediffmail.com 


\title{
Improved Ridge Estimator in Linear Regression with Multicollinearity, Heteroscedastic Errors and Outliers
}

\author{
A. V. Dorugade \\ Y C Mahavidyalaya, Halkarni \\ Maharashtra, India
}

This paper introduces a new estimator, of ridge parameter $k$ for ridge regression and then evaluated by Monte Carlo simulation. We examine the performance of the proposed estimators compared with other well-known estimators for the model with heteroscedastics and/or correlated errors, outlier observations, non-normal errors and suffer from the problem of multicollinearity. It is shown that proposed estimators have a smaller MSE than the ordinary least squared estimator (LS), Hoerl and Kennard (1970) estimator (RR), jackknifed modified ridge (JMR) estimator, and Jackknifed Ridge M-estimator (JRM).

Keywords: Heteroscedasticity, mean squared error, multicollinearity, outlier, ridge estimator

\section{Introduction}

In multiple linear regressions the estimation of parameters is a common interest for many users. It is well known that an LS estimator has been treated as the best unbiased estimator for a long time since it has minimum variance. Multicollinearity, linear or near-linear dependency among the explanatory variables in the regression model, is an important problem faced in applications. If multicollinearity or the ill-conditioned design matrix in linear regression model is present, the LS estimator is sensitive to number 'errors', namely, there is an 'explosion' of the sampling variance of the estimators. Moreover, some of the regression coefficients may be statistically insignificant with wrong sign and meaningful statistical inference becomes impossible for practitioners.

To overcome multicollinearity various biased estimators were put forward in the literature. The Ridge Regression (RR) estimator proposed by Hoerl and

Dr. Dorugade is an Assistant Professor of Statistics. Email him at: adorugade@rediffmail.com. 


\section{A V DORUGADE}

Kennard (1970) is the most popular biased estimator. However, RR estimator has some disadvantages; mainly it is a nonlinear function of the ridge parameter (or biasing constant) $k$. Currently there are various methods for determination and much of the discussions on ridge regression concern the problem of finding or selecting good empirical value of $k$. Our primary aim in this article is overcome this problem by suggesting new estimator for ridge parameter and then evaluate its performance when model defined in linear regression exhibits with not only multicollinearity but also heteroscedastics and/or correlated errors, non-normal errors and outliers, respectively.

Much of the discussions on ridge regression concern the problem of finding better alternative to the LS estimator. Some popular numerical techniques to deal with multicollinearity are the ridge regression due to Singh and Chaubey (1987), Batah, Ramnathan, and Gore (2008), Yang and Chang (2010) and others. Most of the estimation procedures are obtained results when specific assumptions like elements of the random vector $\varepsilon$ were independent and identically distributed random variables are achieved. But if these assumptions are violated, these methods do not assure the desirable results. Involving such problems as heteroscedasticity and autocorrelation few methods including Trenkler (1984), Firinguetti (1989), Bayhan and Bayhan (1998), Özkale (2008), Alheety and Kibria (2009) are available in the present literature. Recently, Li and Yang (2011) suggested Jackknifed Modified Ridge Estimator (JMRE) and show that it superior to the generalized least squares estimate, the generalized modified ridge estimator and the generalized jackknifed ridge estimator, to overcome multicollinearity in the presence of a linear regression model with correlated or heteroscedastic errors.

Apart from the problem of multicollinearity in real life situation, outliers and departure from the normality assumption are common problems in regression. These also produce undesirable effects on the LS estimator. This fact is pointed out by many researchers. Many researchers have pointed out that M-estimator is better than LS estimator in the presence of outliers (Huber, 1981; Rousseeuw \& Leroy, 1987; Birkes \& Dodge, 1993). In standard text like Birkes and Dodge (1993) and Montgomery, Peck, and Vining (2001) have given detail description. Recently, Jadhav and Kashid (2011) gives Jackknifed Ridge M-estimator (JRM) and show that it performs better than LS, ridge and M-estimator in the presence of both outliers and multicollinearity. Hence our secondary aim in this article is to provide an alternative method to combat both the problem of outliers and heteroscedastics and/or correlated errors, respectively in linear regression model in the presence of multicollinearity. 


\section{IMPROVED RIDGE ESTIMATOR IN LINEAR REGRESSION}

\section{Model and Estimators}

Consider, widely used linear regression model

$$
Y=X \beta+\varepsilon
$$

where $Y$ is an $n \times 1$ random vector of response variables, $\boldsymbol{X}$ is a known $n \times p$ matrix with full column rank, $\varepsilon$ is the vector of errors $E(\varepsilon)=0$ and $\operatorname{Cov}(\varepsilon)=\sigma^{2} I n$., $\beta$ is a $p \times 1$ vector of unknown regression parameters and $\sigma^{2}$ is the unknown variance parameter. For the sake of convenience, we assume that the matrix $X$ and response variable $Y$ are standardized in such a way that $X^{\prime} X$ is a non-singular correlation matrix and $X^{\prime} Y$ is the correlation between $X$ and $Y$.

Let $\wedge$ and $T$ be the matrices of eigenvalues and eigenvectors of $X^{\prime} X$,

respectively, satisfying $T^{\prime} X^{\prime} X T=\wedge=\operatorname{diagonal}\left(\lambda_{1}, \lambda_{2}, \ldots, \lambda_{p}\right)$ where $\lambda_{i}$ being the $i^{\text {th }}$ eigenvalue of $X^{\prime} X$ and $T^{\prime} T=T T^{\prime}=I p$. We obtain the equivalent model

$$
Y=Z \alpha+\varepsilon
$$

where $Z=X T$, it implies that $Z^{\prime} Z=\wedge$, and $\alpha=T^{\prime} \beta$ (see Montgomery et al., 2001).

Then LS estimator of $\alpha$ is given by

$$
\hat{\alpha}_{L S}=\left(Z^{\prime} Z\right)^{-1} Z^{\prime} Y=\wedge^{-1} Z^{\prime} Y
$$

Therefore, LS estimator of $\beta$ is given by

$$
\hat{\beta}_{L S}=T \hat{\alpha}_{L S}
$$

\section{Ridge Regression Estimator (RR)}

To overcome multicollinearity under ridge regression, Hoerl and Kennard (1970) suggested an alternative estimate by adding a ridge parameter $k$ to the diagonal elements of the least square estimator. It is given as:

$$
\hat{\alpha}_{R R}=\left\lfloor I-k(\wedge+k I)^{-1}\right\rfloor \hat{\alpha}_{L S}
$$




\section{A V DORUGADE}

Therefore, RR estimator of $\beta$ is given by

$$
\hat{\beta}_{R R}=T \hat{\alpha}_{R R}
$$

and mean square error of $\hat{\alpha}_{R R}$ is

$$
\operatorname{MSE}\left(\hat{\alpha}_{R R}\right)=\hat{\sigma}^{2} \sum_{i=1}^{p} \lambda_{i} /\left(\lambda_{i}+k\right)^{2}+k^{2} \sum_{i=1}^{p} \hat{\alpha}_{i}^{2} /\left(\lambda_{i}+k\right)^{2}
$$

Observe when $k=0$ in (5), MSE of LS estimator of $\alpha$ is recovered. Hence,

$$
\operatorname{MSE}\left(\hat{\alpha}_{L S}\right)=\hat{\sigma}^{2} \sum_{i=1}^{p} 1 / \lambda_{i}
$$

\section{Jackknifed Modified Ridge Estimator (JMR)}

Li and Yang (2011) introduced a jackknifed modified ridge (JMR) estimator an alternative method to overcome multicollinearity in the presence of a linear regression model with correlated or heteroscedastic errors. With the assumptions $E(\varepsilon)=0$ and $\operatorname{Cov}(\varepsilon)=\sigma^{2} V$, where $V$ is a known $n \times n$ symmetric positive definite (pd) matrix there exists a nonsingular symmetric matrix $P$ such that $V^{-1}=P^{\prime} P$ and $\sigma^{2}>0$ is the unknown variance parameter. Then the linear model given in (2) can be written as

$$
\tilde{Y}=\tilde{Z} \alpha+\tilde{\varepsilon}
$$

where, $\tilde{Y}=P Y, \tilde{\varepsilon}=P \varepsilon$ and $\tilde{Z}=P Z$ with a prior mean $c=\sum_{i=1}^{p} \hat{\alpha}_{i L S} / p$ and $k>0$ is the ridge parameter (see Li \& Yang, 2011). The JMR estimator of $\alpha$ is given as

$$
\begin{gathered}
\hat{\alpha}_{J M R}=\left\lfloor I-k^{2}\left(\tilde{Z}^{\prime} \tilde{Z}+k I\right)^{-2}\right\rfloor\left(\tilde{Z}^{\prime} \tilde{Z}\right)^{-1} \tilde{Z}^{\prime} \tilde{Y}+k^{2}\left(\tilde{Z}^{\prime} \tilde{Z}+k I\right)^{-2} c \\
\hat{\beta}_{J M R}=T \hat{\alpha}_{J M R}
\end{gathered}
$$




\section{IMPROVED RIDGE ESTIMATOR IN LINEAR REGRESSION}

Hoerl, Kennard, and Baldwin (1975) suggested the value of ' $k$ ' should be chosen small enough so the mean squared error of ridge estimator is less than the mean squared error of LS estimator. Among the various methods here, the ridge parameter was used to compute $\hat{\alpha}_{R R}$ and $\hat{\alpha}_{J M R}$ given by Hoerl et al. (1975), given as

$$
k_{H K B}=p \hat{\sigma}^{2} / \sum_{i=1}^{p} \hat{\alpha}_{i}^{2}
$$

where $\hat{\alpha}_{i}$ is the $i^{\text {th }}$ element of $\hat{\alpha}_{L S}, i=1,2, \ldots, p$ and $\hat{\sigma}^{2}$ is the LS estimator of $\sigma^{2}$ i.e.

$$
\hat{\sigma}^{2}=\frac{Y^{\prime} Y-\hat{\alpha}_{L S}^{\prime} Z^{\prime} Y}{n-p-1} .
$$

\section{Jackknifed Ridge M-Estimator (JRM)}

Jadhav and Kashid (2011) gave Jackknifed Ridge M-estimator (JRM) which takes into account the presence of both multicollinearity and outlier problems simultaneously.

It is given as:

$$
\hat{\alpha}_{J R M}=\left(I-k^{* 2} A^{-2}\right) \hat{\alpha}_{M}
$$

Where, $\hat{\alpha}_{M}$ is an M-estimator of $\alpha$, which is obtained by solving the following equations

$$
\sum_{i=1}^{n} v_{i j} \psi\left(Y_{i}-z_{i}^{\prime} \hat{\alpha}_{M}\right)=0
$$

where $\psi($.$) is some function (see Huber (1981), Hampel, Ronchetti, Rousseeuw,$ and Stahel (1986)). Therefore, JRM estimator of $\beta$ is given by

$$
\hat{\beta}_{J R M}=T \hat{\alpha}_{J R M}
$$




\section{A V DORUGADE}

where, ridge parameter says $k^{*}$ can be estimated using robust estimates of $\sigma^{2}$ and $\alpha$ respectively given by $s$ and $\hat{\alpha}_{M}$, it is obtained by

$$
k^{*}=\frac{p s^{2}}{\hat{\alpha}_{M}^{\prime} \hat{\alpha}_{M}},
$$

where $s=1.4826$ median $\mid e_{i}-$ median $\left(e_{1}\right) \mid$ and $e_{i}$ is $i^{\text {th }}$ residual obtained by using LS estimator.

\section{Proposed Ridge Parameter}

The existence of multicollinearity may cause to have wide confidence interval for individual parameters or linear combination of the parameters, may give estimates with wrong signs. Ridge regression is a concept proposed in the sixties to combat the multicollinearity in regression problems. After then, many new versions of this method have been studied to extended Hoerl and Kennard (1970) original estimator. It has been made a more definite comparison of these various versions of the biased estimators versus the unbiased LS estimator. The constant, $k>0$ is known as ridge parameter which plays an important role in ridge regression. As $k$ increases from 0 and continues upto $\infty$ the regression estimates tend towards 0 . In ridge regression our interest lies in finding a value of $k$ such that the reduction in the variance term is greater than the increase in the squared bias. Though these estimators result in biased, for certain value of $k$, they yield minimum mean squared error (MMSE) compared to the LS estimator (see Hoerl \& Kennard, 1970). In the last decades, researchers concentrated on estimating the shrinkage ridge parameter $k$ in different ways and under different situations, and then compared the results with those obtained by applying the LS estimators. Much of the discussions on ridge regression concern the problem of finding good empirical value of $k$.

Ridge regression estimator of Hoerl and Kennard (1970) was proposed as alternative to the least squares estimator in the presence of multicollinearity. It depends on the biasing parameter $k$ which is the Lagrange multiplier used in the objective function although proposing the estimator. To compute the ridge regression estimator, the analyst must know the value of $k$. Therefore, various estimators of $k$ were proposed. Many different techniques for estimating $k$ have been proposed or suggested by different researchers Hoerl et al. (1975), Lawless and Wang (1976), Kibria (2003), Khalaf and Shukur (2005), Alkhamisi and 


\section{IMPROVED RIDGE ESTIMATOR IN LINEAR REGRESSION}

Shukur (2007), Muniz and Kibria (2009), Dorugade and Kashid (2010), Al-Hassan (2010), Muniz, Kibria, Mansson, and Shukur (2012) to mention a few. The well known of them is obtained by minimizing the mean square error of the ridge regression estimator and it depends on the variance of the regression model, $\sigma^{2}$, and the parameter vector $\beta$. Since $\sigma^{2}$ and $\beta$ are unknown, the analyst have to use estimates of these parameters. In this article we propose estimator of $k$ depends on the variance of the regression model, $\sigma^{2}$ only. Even though this approach is quite straightforward and simple, to the best of our knowledge, it has not been considered in the literature at all.

We denote our ridge parameter by $k_{R}$ and given by

$$
k_{R}=\sigma,
$$

where error variance $\sigma^{2}$, replaced by its LS estimator $\hat{\sigma}^{2}$ i.e.

$$
\hat{\sigma}^{2}=\frac{Y^{\prime} Y-\hat{\alpha}_{L S}^{\prime} Z^{\prime} Y}{n-p-1}
$$

The RR estimator based on $k_{R}$ is given as

$$
\hat{\alpha}_{R R}^{*}=\left\lfloor I-k_{R}\left(\wedge+k_{R} I\right)^{-1}\right\rfloor \hat{\alpha}_{L S} .
$$

Therefore, $\mathrm{RR}$ estimator of $\beta$ is given by

$$
\hat{\beta}_{R R}^{*}=T \hat{\alpha}_{R R}^{*}
$$

and using (5) mean square error of $\hat{\alpha}_{R R}^{*}$ is

$$
\operatorname{MSE}\left(\hat{\alpha}_{R R}^{*}\right)=\frac{\hat{\sigma}^{2}}{\left(\lambda_{i}+\hat{\sigma}\right)^{2}} \sum_{i=1}^{p}\left(\lambda_{i}+\hat{\alpha}_{i}^{2}\right)
$$

\section{Comparison Between the $\hat{\alpha}_{R R}^{*}$ and $\hat{\alpha}_{R R}$}

Using (5) and (13), consider the following difference 


\section{A V DORUGADE}

$$
\begin{aligned}
\operatorname{MSE}\left(\hat{\alpha}_{R R}\right)-\operatorname{MSE}\left(\hat{\alpha}_{R R}^{*}\right) & =\sum_{i=1}^{p}\left[\frac{\left(\hat{\sigma}^{2} \lambda_{i}+k^{2} \hat{\alpha}_{i}^{2}\right)}{\left(\lambda_{i}+k\right)^{2}}\right]-\hat{\sigma}^{2} \sum_{i=1}^{p}\left[\frac{\left(\lambda_{i}+\hat{\alpha}_{i}^{2}\right)}{\left(\lambda_{i}+\hat{\sigma}\right)^{2}}\right] \\
& =\sum_{i=1}^{p} \frac{\left(k^{2} \hat{\alpha}_{i}^{2}\right)}{\left(\lambda_{i}+k\right)^{2}}+\hat{\sigma}^{2} \sum_{i=1}^{p}\left[\frac{\left(\lambda_{i}+\hat{\sigma}\right)^{2} \lambda_{i}-\left(\lambda_{i}+k\right)^{2}\left(\lambda_{i}+\hat{\alpha}_{i}^{2}\right)}{\left(\lambda_{i}+k\right)^{2}\left(\lambda_{i}+\hat{\sigma}\right)^{2}}\right]
\end{aligned}
$$

From above equation, the difference $\operatorname{MSE}\left(\hat{\alpha}_{R R}\right)-\operatorname{MSE}\left(\hat{\alpha}_{R R}^{*}\right)$ can be positive because

$$
\sum_{i=1}^{p}\left(\lambda_{i}+\hat{\sigma}\right)^{2} \lambda_{i} \geq \sum_{i=1}^{p}\left(\lambda_{i}+k\right)^{2}\left(\lambda_{i}+\hat{\alpha}_{i}^{2}\right)
$$

Thus, $\operatorname{MSE}\left(\hat{\alpha}_{R R}\right) \geq \operatorname{MSE}\left(\hat{\alpha}_{R R}^{*}\right)$

\section{Simulation Study}

Consider the behavior of the proposed parameter estimators via a simulation study. Most of the researchers compare the performance of their suggested ridge parameter in the sense of smaller MSE compared to LS and other well-known existing ridge parameters via ridge regression estimators. But, we evaluate the performance of our suggested ridge parameter by considering following different situations in linear regression when data exhibits with multicollinearity.

Case I. Data generated using normal errors.

Case II. Data generated using heteroscedastic errors.

Case III. Data generated using outlier observations.

Case IV. Data generated using outlier observations and heteroscedastic errors.

Case V. Data generated using non-normal errors.

Consider the average MSE (AMSE) of the $\hat{\beta}_{L S}, \hat{\beta}_{R R}, \hat{\beta}_{J M R}, \hat{\beta}_{J R M}$ and $\hat{\beta}_{R R}^{*}$ estimators for different degrees of multicollinearity. We consider the true model as $Y=X \beta+\varepsilon$. Following McDonald and Galarneau (1975) the explanatory variables are generated by 


\section{IMPROVED RIDGE ESTIMATOR IN LINEAR REGRESSION}

$$
x_{i j}=\left(1-\rho^{2}\right)^{1 / 2} u_{i j}+\rho u_{i p}, \quad i=1,2, \ldots, n \quad j=1,2, \ldots, p
$$

where $u i j$ are independent standard normal pseudo-random numbers and $\rho$ is specified so that the theoretical correlation between any two explanatory variables is given by $\rho 2$. In this study, to investigate the effects of different degrees of multicollinearity on the estimators, we consider two different correlations, $\rho=0.85,0.90,0.95$ and 0.99 . $\beta$ parameter vectors are chosen arbitrarily for $p=4$, respectively. We assumed samples of size of 25,60 and 100 . Estimators $\hat{\beta}_{L S}, \hat{\beta}_{R R}$, $\hat{\beta}_{J M R}, \hat{\beta}_{J R M}$ and $\hat{\beta}_{R R}^{*}$ are computed and obtained the average MSE (AMSE) of estimators. The experiment is repeated 1500 times using the following expression.

$$
\operatorname{AMSE}(\hat{\beta})=\frac{1}{1500} \sum_{i=1}^{p} \sum_{j=1}^{1500}\left(\hat{\beta}_{i j}-\beta_{i}\right)^{2}
$$

where, $\hat{\beta}_{i j}$ denote the estimator of the $i^{\text {th }}$ parameter in the $j^{\text {th }}$ replication and $\beta_{i}$, $i=1,2, \ldots, p$ are the true parameter values. Consider the method that leads to the minimum AMSE to the best from the MSE point of view.

Case I. Here $\varepsilon$ follows a normal distribution $N\left(0, \sigma^{2} I_{n}\right)$. The variance of the error terms is taken as $\sigma^{2}=1,5,10$ and 25. Firstly, we computed the AMSE values for $\hat{\beta}_{L S}, \hat{\beta}_{R R}, \hat{\beta}_{J M R}, \hat{\beta}_{J R M}$ and $\hat{\beta}_{R R}^{*}$ for various values of triplet $\left(\rho, n, \sigma^{2}\right)$ for $p=4$ and reported in Table 1 . 


\section{A V DORUGADE}

Table 1. AMSE of LS and various ridge estimators $\left(p=4\right.$ and $\left.\beta=(5,3,4,2)^{\prime}\right)$

\begin{tabular}{|c|c|c|c|c|c|c|c|c|c|}
\hline \multirow[b]{2}{*}{$\rho$} & \multirow{2}{*}{ Estimator } & \multicolumn{4}{|c|}{$n=25$} & \multicolumn{4}{|c|}{100} \\
\hline & & $\sigma^{2}=1$ & 5 & 10 & 25 & 1 & 5 & 10 & 25 \\
\hline \multirow{5}{*}{0.85} & $\hat{\beta}_{L S}$ & 3.38 & 93.73 & 394.86 & 2451.82 & 0.59 & 12.36 & 46.85 & 297.86 \\
\hline & $\hat{\beta}_{R R}$ & 2.77 & 33.84 & 125.85 & 726.46 & 0.57 & 7.86 & 20.38 & 98.82 \\
\hline & $\hat{\beta}_{\text {JMR }}$ & 2.50 & 45.32 & 203.00 & 1202.44 & 0.68 & 7.34 & 24.22 & 152.46 \\
\hline & $\hat{\beta}_{J R M}$ & 3.00 & 50.99 & 192.95 & 1146.93 & 0.58 & 9.87 & 28.98 & 153.65 \\
\hline & $\hat{\beta}_{R R}^{*}$ & 2.03 & 14.21 & 34.35 & 138.08 & 0.50 & 5.27 & 12.93 & 54.74 \\
\hline \multirow{5}{*}{0.90} & $\hat{\beta}_{L S}$ & 19.20 & 526.30 & 1694.80 & 12498.50 & 2.90 & 90.30 & 281.50 & 2202.30 \\
\hline & $\hat{\beta}_{R R}$ & 10.66 & 172.23 & 468.04 & 3571.36 & 2.54 & 37.88 & 86.29 & 717.45 \\
\hline & $\hat{\beta}_{J M R}$ & 11.01 & 273.95 & 810.47 & 6158.55 & 2.49 & 47.82 & 132.76 & 1196.13 \\
\hline & $\hat{\beta}_{J R M}$ & 13.65 & 241.76 & 740.49 & 5369.88 & 2.78 & 52.35 & 140.80 & 1046.13 \\
\hline & $\hat{\beta}_{R R}^{*}$ & 4.14 & 12.58 & 22.72 & 82.23 & 1.80 & 8.29 & 11.38 & 38.41 \\
\hline \multirow{5}{*}{0.95} & $\hat{\beta}_{L S}$ & 163.00 & 5120.00 & 19151.00 & $\begin{array}{r}129866.0 \\
0\end{array}$ & 31.00 & 968.00 & 3581.00 & 17759.00 \\
\hline & $\hat{\beta}_{R R}$ & 47.40 & 1659.60 & 4884.20 & 34858.20 & 15.20 & 308.10 & 1047.60 & 4582.80 \\
\hline & $\hat{\beta}_{J M R}$ & 68.60 & 2273.70 & 7997.70 & 51354.00 & 19.80 & 447.40 & 1576.00 & 7899.10 \\
\hline & $\hat{\beta}_{J R M}$ & 74.20 & 2619.40 & 8863.60 & 65176.40 & 16.50 & 507.60 & 1777.80 & 8105.60 \\
\hline & $\hat{\beta}_{R R}^{*}$ & 4.72 & 7.17 & 12.51 & 49.62 & 4.03 & 5.98 & 6.76 & 17.48 \\
\hline \multirow{5}{*}{0.99} & $\hat{\beta}_{L S}$ & 1804.00 & 48622.00 & $\begin{array}{r}220824.0 \\
0\end{array}$ & $\begin{array}{r}1400590 . \\
00\end{array}$ & 320.00 & 7592.00 & 30461.00 & $\begin{array}{r}213251.0 \\
0\end{array}$ \\
\hline & $\hat{\beta}_{R R}$ & 470.00 & 14008.00 & 76100.00 & $\begin{array}{r}387524.0 \\
0\end{array}$ & 84.00 & 2015.00 & 8000.00 & 61836.00 \\
\hline & $\hat{\beta}_{J M R}$ & 717.00 & 21406.00 & $\begin{array}{r}105437.0 \\
0\end{array}$ & $\begin{array}{r}573785.0 \\
0\end{array}$ & 144.00 & 3306.00 & 12875.00 & 90558.00 \\
\hline & $\hat{\beta}_{J R M}$ & 840.00 & 23485.00 & $\begin{array}{r}119862.0 \\
0\end{array}$ & $\begin{array}{r}722312.0 \\
0\end{array}$ & 141.00 & 3514.00 & 14124.00 & $\begin{array}{r}104016.0 \\
0\end{array}$ \\
\hline & $\hat{\beta}_{R R}^{*}$ & 5.05 & 6.94 & 10.52 & 47.23 & 4.93 & 5.28 & 6.75 & 13.24 \\
\hline
\end{tabular}

From Table 1, we observe that performance of our proposed estimator $\hat{\beta}_{R R}^{*}$ is better than $\hat{\beta}_{L S}, \hat{\beta}_{R R}, \hat{\beta}_{J M R}$ and $\hat{\beta}_{J R M}$ for various values of triplet $\left(\rho, n, \sigma^{2}\right)$. Because $\hat{\beta}_{R R}, \hat{\beta}_{J M R}$ gives better performance than $\hat{\beta}_{L S}$ and $\hat{\beta}_{J R M}$ for various values of triplet $\left(\rho, n, \sigma^{2}\right)$. Particularly for increasing degree of multicollinearity, $\hat{\beta}_{R R}^{*}$ gives significantly smaller AMSE values as compare to other estimators. 


\section{IMPROVED RIDGE ESTIMATOR IN LINEAR REGRESSION}

Case II. Consider the problem of heteroscedasticity. Assume that the elements of the random vector $\varepsilon$ were not independent and identically distributed random variables. To introduce heteroscedastics and/or correlated errors in the model given in (2) and converted into model given in (7) matrix $V$ will be estimated by method suggested by Firinguetti (1989). In the present study we choose $\rho=0.95$ and consider matrix $V$ is estimated as below

Table 2. AMSE of LS and various ridge estimators $\left(p=4\right.$ and $\left.\beta=(5,7,3,1)^{\prime}\right)$

\begin{tabular}{|c|c|c|c|c|c|c|c|c|c|}
\hline \multirow{2}{*}{$\rho$} & \multirow{2}{*}{ Estimator } & \multicolumn{4}{|c|}{$n=25$} & \multicolumn{4}{|c|}{100} \\
\hline & & $\sigma^{2}=1$ & 5 & 10 & 25 & 1 & 5 & 10 & 25 \\
\hline \multirow{5}{*}{0.85} & $\hat{\beta}_{L S}$ & 20.10 & 596.90 & 2180.80 & 12215.10 & 8.60 & 297.80 & 928.00 & 6097.90 \\
\hline & $\hat{\beta}_{R R}$ & 17.31 & 451.55 & 1648.97 & 9146.44 & 8.48 & 264.17 & 802.37 & 5347.04 \\
\hline & $\hat{\beta}_{J M R}$ & 18.34 & 481.14 & 1786.08 & 9709.46 & 8.53 & 270.53 & 835.75 & 5524.28 \\
\hline & $\hat{\beta}_{J R M}$ & 17.30 & 501.90 & 1819.30 & 10198.20 & 8.83 & 275.20 & 846.90 & 5609.70 \\
\hline & $\hat{\beta}_{R R}^{*}$ & 13.62 & 333.51 & 1186.55 & 6739.84 & 7.40 & 181.94 & 568.38 & 3582.63 \\
\hline \multirow{5}{*}{0.90} & $\hat{\beta}_{L S}$ & 78.80 & 1723.70 & 6451.40 & 46119.30 & 26.60 & 693.50 & 2406.00 & 18390.80 \\
\hline & $\hat{\beta}_{R R}$ & 55.80 & 1002.10 & 3739.50 & 27348.40 & 24.20 & 545.10 & 1839.00 & 14318.50 \\
\hline & $\hat{\beta}_{J M R}$ & 61.50 & 1139.30 & 4336.70 & 32280.90 & 24.70 & 580.30 & 1974.20 & 15252.90 \\
\hline & $\hat{\beta}_{J R M}$ & 61.80 & 1263.50 & 4713.30 & 34289.70 & 26.80 & 607.50 & 2074.60 & 16102.30 \\
\hline & $\hat{\beta}_{R R}^{*}$ & 20.50 & 334.41 & 1069.86 & 7534.85 & 12.05 & 153.74 & 695.38 & 4277.38 \\
\hline \multirow{5}{*}{0.95} & $\hat{\beta}_{L S}$ & 669.00 & 15446.00 & 68939.00 & 393609.00 & 176.00 & 4798.00 & 20237.00 & 135777.00 \\
\hline & $\hat{\beta}_{R R}$ & 378.00 & 8964.00 & 37083.00 & 216822.00 & 130.00 & 3306.00 & 13954.00 & 95154.00 \\
\hline & $\hat{\beta}_{J M R}$ & 448.00 & 10472.00 & 43461.00 & 255218.00 & 141.00 & 3630.00 & 15264.00 & 105690.00 \\
\hline & $\hat{\beta}_{J R M}$ & 490.00 & 11207.00 & 50079.00 & 279251.00 & 142.00 & 3893.00 & 16606.00 & 112258.00 \\
\hline & $\hat{\beta}_{R R}^{*}$ & 21.14 & 339.32 & 1065.06 & 8295.32 & 12.41 & 125.99 & 492.23 & 2897.20 \\
\hline \multirow{5}{*}{0.99} & $\hat{\beta}_{L S}$ & 5874.00 & 156068.00 & 599010.00 & 4373664.00 & 2221.00 & 56796.00 & 240541.00 & 1323948.00 \\
\hline & $\hat{\beta}_{R R}$ & 3093.00 & 77901.00 & 333494.00 & 2335520.00 & 1635.00 & 41391.00 & 164114.00 & 958398.00 \\
\hline & $\hat{\beta}_{I M R}$ & 3773.00 & 93485.00 & 394200.00 & 2770590.00 & 1777.00 & 45134.00 & 185561.00 & 1038487.00 \\
\hline & $\hat{\beta}_{J R M}$ & 4072.00 & 106393.00 & 441980.00 & 3111729.00 & 1881.00 & 48157.00 & 198360.00 & 1115227.00 \\
\hline & $\hat{\beta}_{R R}^{*}$ & 17.44 & 277.09 & 1282.84 & 6875.21 & 9.27 & 143.95 & 531.16 & 3250.81 \\
\hline
\end{tabular}




\section{A V DORUGADE}

$$
V=\frac{1}{1-\rho^{2}}\left[\begin{array}{cccc}
1 & \rho & \cdots & \rho^{n-1} \\
\rho & 1 & \cdots & \rho^{n-2} \\
\cdot & \cdot & \cdot & \cdot \\
\cdot & \cdot & \cdot & \cdot \\
\cdot & \cdot & \cdot & \cdot \\
\rho^{n-1} & \rho^{n-2} & \cdots & 1
\end{array}\right]
$$

The AMSE was computed for various values of combination $\left(\rho, n, V, \sigma^{2}\right)$ for $p=4$ and reported in

From the results reported in Table 2 , observe $\hat{\beta}_{R R}, \hat{\beta}_{J M R}$ gives equivalently better performance than $\hat{\beta}_{L S}$ and $\hat{\beta}_{J R M}$ for various values of triplet $\left(\rho, n, \sigma^{2}\right)$. However performance of $\hat{\beta}_{R R}^{*}$ is better than $\hat{\beta}_{L S}, \hat{\beta}_{R R}, \hat{\beta}_{J M R}$ and $\hat{\beta}_{J R M}$ for various values of triplet $\left(\rho, n, \sigma^{2}\right)$.

Case III. The same simulation experiment is repeated for 1500 times for all combinations of $\rho, n$, and $\sigma^{2}$ by introducing one and two outliers for different model specifications. For $p=4$ we computed the AMSE for various values of combination $\left(\rho, n, V, \sigma^{2}\right)$ for one and two outliers and reported in Table 3 and 4 respectively.

Results in Table 3 and 4 , shows that $\hat{\beta}_{J R M}$ gives better performance than $\hat{\beta}_{L S}, \hat{\beta}_{R R}$ and $\hat{\beta}_{J M R}$ for all combinations of $\rho, n$, and $\sigma^{2}$. However, proposed estimator $\hat{\beta}_{R R}^{*}$ gives better performance than $\hat{\beta}_{L S}, \hat{\beta}_{R R}$ and $\hat{\beta}_{J M R}$ including $\hat{\beta}_{J R M}$ for various values of triplet $\left(\rho, n, \sigma^{2}\right)$.

Case IV. With respect to our secondary aim of the proposed work in this article, here we evaluate the performance of proposed estimator $\hat{\beta}_{R R}^{*}$ against $\hat{\beta}_{L S}$, $\hat{\beta}_{R R}, \hat{\alpha}_{J M R}$ and $\hat{\beta}_{J R M}$ for the simulated data exits with one or multiple outliers and heteroscedastics and/or correlated errors, in linear regression model in the presence of multicollinearity. We introduce respectively one and two outliers in the simulated data with heteroscedastics and/or correlated errors, where heteroscedastics and/or correlated errors are introduced using the same method as given in Case II with the help of matrix $V$ at $\rho=0.95$. The simulation experiment is repeated for 1500 times for all combinations of $\rho, n, \sigma^{2}$ and $V$ and computed 


\section{IMPROVED RIDGE ESTIMATOR IN LINEAR REGRESSION}

AMSE values for one and two outliers respectively, for $p=4$ are reported in Tables 5 and 6.

Table 3. AMSE of LS and various ridge estimators (With one outlier, $p=4$ and $\left.\beta=(10,3,4,1)^{\prime}\right)$

\begin{tabular}{|c|c|c|c|c|c|c|c|c|c|}
\hline \multirow[b]{2}{*}{$\rho$} & \multirow{2}{*}{ Estimator } & \multicolumn{4}{|c|}{$n=25$} & \multicolumn{4}{|c|}{100} \\
\hline & & $\sigma^{2}=1$ & 5 & 10 & 25 & 1 & 5 & 10 & 25 \\
\hline \multirow{5}{*}{0.85} & $\hat{\beta}_{L S}$ & 63093.50 & 51479.90 & 56809.70 & 52816.70 & 1780.00 & 1744.00 & 1845.80 & 2164.60 \\
\hline & $\hat{\beta}_{R R}$ & 19398.90 & 13650.00 & 16861.10 & 14144.10 & 510.70 & 519.20 & 540.70 & 569.40 \\
\hline & $\hat{\beta}_{J M R}$ & 28805.10 & 22122.40 & 28174.90 & 22578.70 & 858.20 & 861.20 & 864.90 & 951.70 \\
\hline & $\hat{\beta}_{J R M}$ & 6261.57 & 4513.43 & 5729.10 & 4750.19 & 159.57 & 167.09 & 177.22 & 174.30 \\
\hline & $\hat{\beta}_{R R}^{*}$ & 3544.36 & 3922.95 & 4084.13 & 3994.31 & 299.85 & 268.90 & 283.91 & 283.74 \\
\hline \multirow{5}{*}{0.90} & $\hat{\beta}_{L S}$ & 324191.00 & 308106.00 & 304710.00 & 335486.00 & 9945.00 & 9611.00 & 9304.00 & 11664.00 \\
\hline & $\hat{\beta}_{R R}$ & 95866.80 & 91106.00 & 78611.80 & 90283.00 & 2893.40 & 2593.80 & 2465.90 & 3214.60 \\
\hline & $\hat{\beta}_{J M R}$ & 146348.00 & 138431.00 & 119726.00 & 138150.00 & 4469.00 & 4023.00 & 3873.00 & 4911.00 \\
\hline & $\hat{\beta}_{J R M}$ & 28132.50 & 25922.10 & 20062.80 & 24722.10 & 768.80 & 636.50 & 603.70 & 873.00 \\
\hline & $\hat{\beta}_{R R}^{*}$ & 2163.99 & 2379.11 & 1851.32 & 2123.19 & 165.03 & 169.74 & 171.49 & 208.08 \\
\hline \multirow{5}{*}{0.95} & $\hat{\beta}_{L S}$ & 3563590.00 & 3754264.00 & 3331604.00 & 3317982.00 & 101439.00 & 109183.00 & 102304.00 & 129443.00 \\
\hline & $\hat{\beta}_{R R}$ & 1013175.00 & 1025041.00 & 818990.00 & 941995.00 & 26462.00 & 30554.00 & 25880.00 & 38298.00 \\
\hline & $\hat{\beta}_{J M R}$ & 1569621.00 & 1638275.00 & 1360205.00 & 1409372.00 & 41486.00 & 45617.00 & 39268.00 & 58030.00 \\
\hline & $\hat{\beta}_{J R M}$ & 277101.00 & 276193.00 & 214808.00 & 251716.00 & 6206.00 & 7271.00 & 6163.00 & 10128.00 \\
\hline & $\hat{\beta}_{R R}^{*}$ & 1244.35 & 1248.52 & 1174.22 & 1319.63 & 65.37 & 62.37 & 63.64 & 75.89 \\
\hline \multirow{5}{*}{0.99} & $\hat{\beta}_{L S}$ & 36409135.00 & 33502778.00 & 28002957.00 & 33771993.00 & 1177860.00 & 1021218.00 & 968594.00 & 1107336.00 \\
\hline & $\hat{\beta}_{R R}$ & 9715560.00 & 8705724.00 & 6824202.00 & 8129113.00 & 295078.00 & 249096.00 & 230598.00 & 295623.00 \\
\hline & $\hat{\beta}_{J M R}$ & 15072802.00 & 13295260.00 & 10408403.00 & 12343872.00 & 473153.00 & 397830.00 & 375101.00 & 482420.00 \\
\hline & $\hat{\beta}_{J R M}$ & 2534630.00 & 2280994.00 & 1725382.00 & 1839086.00 & 62366.00 & 53576.00 & 50324.00 & 71652.00 \\
\hline & $\hat{\beta}_{R R}^{*}$ & 1121.53 & 1109.93 & 1258.06 & 1247.55 & 50.70 & 47.03 & 48.06 & 59.10 \\
\hline
\end{tabular}




\section{A V DORUGADE}

Table 4. AMSE of LS and various ridge estimators (With two outlier, $p=4$ and $\left.\beta=(3,3,8,1)^{\prime}\right)$

\begin{tabular}{|c|c|c|c|c|c|c|c|c|c|}
\hline \multirow{2}{*}{$\rho$} & \multirow{2}{*}{ Estimator } & \multicolumn{4}{|c|}{$n=25$} & \multicolumn{4}{|c|}{100} \\
\hline & & $\sigma^{2}=1$ & 5 & 10 & 25 & 1 & 5 & 10 & 25 \\
\hline \multirow{5}{*}{0.85} & $\hat{\beta}_{L S}$ & 96023.00 & 103032.00 & 140414.00 & 114534.00 & 3048.00 & 3267.00 & 3679.00 & 3899.00 \\
\hline & $\hat{\beta}_{R R}$ & 29822.20 & 31245.50 & 51089.80 & 34497.80 & 916.70 & 1170.50 & 1264.30 & 1230.90 \\
\hline & $\hat{\beta}_{J M R}$ & 46684.60 & 50507.30 & 71186.90 & 51338.00 & 1479.80 & 1809.00 & 1828.50 & 1904.30 \\
\hline & $\hat{\beta}_{J R M}$ & 11177.50 & 10682.10 & 19345.00 & 12368.40 & 329.40 & 463.90 & 454.20 & 431.70 \\
\hline & $\hat{\beta}_{R R}^{*}$ & 6494.94 & 7297.01 & 8536.97 & 7910.10 & 493.91 & 571.06 & 563.61 & 626.35 \\
\hline \multirow{5}{*}{0.90} & $\hat{\beta}_{L S}$ & 589009.00 & 649515.00 & 531514.00 & 565691.00 & 16516.00 & 15254.00 & 17831.00 & 20356.00 \\
\hline & $\hat{\beta}_{R R}$ & 170930.00 & 201753.00 & 154311.00 & 162987.00 & 4617.00 & 3778.00 & 4867.00 & 5477.00 \\
\hline & $\hat{\beta}_{J M R}$ & 265753.00 & 297287.00 & 221441.00 & 254970.00 & 7301.00 & 6493.00 & 7623.00 & 8377.00 \\
\hline & $\hat{\beta}_{J R M}$ & 47232.60 & 54401.30 & 46217.10 & 43368.10 & 1252.50 & 956.60 & 1282.50 & 1436.80 \\
\hline & $\hat{\beta}_{R R}^{*}$ & 5099.35 & 4651.04 & 4997.34 & 4731.34 & 340.98 & 329.05 & 324.26 & 354.44 \\
\hline \multirow{5}{*}{0.95} & $\hat{\beta}_{L S}$ & 6581935.00 & 6082993.00 & 6188108.00 & 5363832.00 & 188136.00 & 201837.00 & 167767.00 & 193081.00 \\
\hline & $\hat{\beta}_{R R}$ & 1936566.00 & 1645660.00 & 1869023.00 & 1084286.00 & 51291.00 & 58167.00 & 37254.00 & 48946.00 \\
\hline & $\hat{\beta}_{J M R}$ & 2791415.00 & 2505398.00 & 2695831.00 & 1824673.00 & 76196.00 & 90786.00 & 68680.00 & 78656.00 \\
\hline & $\hat{\beta}_{J R M}$ & 510121.00 & 462091.00 & 545257.00 & 221990.00 & 12362.00 & 15340.00 & 8067.00 & 11273.00 \\
\hline & $\hat{\beta}_{R R}^{*}$ & 3329.23 & 3610.92 & 3343.09 & 3315.94 & 160.60 & 164.00 & 159.19 & 169.48 \\
\hline \multirow{5}{*}{0.99} & $\hat{\beta}_{L S}$ & 57371428.00 & 59252077.00 & 56364293.00 & 63635854.00 & 1909329.00 & 1609903.00 & 2052240.00 & 2219103.00 \\
\hline & $\hat{\beta}_{R R}$ & 14545081.00 & 16779217.00 & 15804173.00 & 16298348.00 & 466721.00 & 354639.00 & 503548.00 & 615258.00 \\
\hline & $\hat{\beta}_{J M R}$ & 23238145.00 & 25256656.00 & 25038333.00 & 25397750.00 & 733936.00 & 639763.00 & 798444.00 & 963890.00 \\
\hline & $\hat{\beta}_{J R M}$ & 3842991.00 & 4420227.00 & 4316709.00 & 4018464.00 & 106047.00 & 76237.00 & 108351.00 & 145024.00 \\
\hline & $\hat{\beta}_{R R}^{*}$ & 3300.96 & 3350.57 & 3304.98 & 3206.13 & 135.45 & 134.47 & 140.39 & 154.87 \\
\hline
\end{tabular}




\section{IMPROVED RIDGE ESTIMATOR IN LINEAR REGRESSION}

Table 5. AMSE of LS and various ridge estimators (With one outlier, $p=4$ and $\left.\beta=(7,3,1,2)^{\prime}\right)$

\begin{tabular}{|c|c|c|c|c|c|c|c|c|c|}
\hline \multirow{2}{*}{$\rho$} & \multirow{2}{*}{ Estimator } & \multicolumn{4}{|c|}{$n=25$} & \multicolumn{4}{|c|}{100} \\
\hline & & $\sigma^{2}=1$ & 5 & 10 & 25 & 1 & 5 & 10 & 25 \\
\hline \multirow{5}{*}{0.85} & $\hat{\beta}_{L S}$ & 9600.90 & 8815.80 & 10990.80 & 18945.10 & 164.90 & 382.60 & 1120.40 & 6778.00 \\
\hline & $\hat{\beta}_{R R}$ & 3220.50 & 3195.20 & 4573.30 & 10069.10 & 92.40 & 279.70 & 881.30 & 5778.20 \\
\hline & $\hat{\beta}_{J M R}$ & 4746.10 & 4343.20 & 5937.50 & 11893.60 & 108.80 & 305.20 & 937.50 & 6059.80 \\
\hline & $\hat{\beta}_{J R M}$ & 1690.05 & 1820.80 & 2773.62 & 7195.41 & 61.33 & 225.06 & 673.48 & 4722.61 \\
\hline & $\hat{\beta}_{R R}^{*}$ & 1325.28 & 1417.28 & 2279.12 & 6087.94 & 66.49 & 218.87 & 580.02 & 3822.50 \\
\hline \multirow{5}{*}{0.90} & $\hat{\beta}_{L S}$ & 30584.90 & 33646.90 & 44180.40 & 69210.60 & 391.30 & 1197.60 & 2950.30 & 19979.50 \\
\hline & $\hat{\beta}_{R R}$ & 8051.80 & 9919.30 & 13989.70 & 27391.40 & 116.60 & 632.70 & 1839.80 & 15375.60 \\
\hline & $\hat{\beta}_{I M R}$ & 11883.10 & 14706.20 & 19874.50 & 35823.80 & 171.30 & 738.40 & 2094.40 & 16489.40 \\
\hline & $\hat{\beta}_{J R M}$ & 2702.40 & 3636.80 & 5393.60 & 13457.10 & 48.60 & 335.00 & 1032.20 & 10209.30 \\
\hline & $\hat{\beta}_{R R}^{*}$ & 897.59 & 1639.73 & 2314.36 & 6054.40 & 39.43 & 167.23 & 444.88 & 3598.33 \\
\hline \multirow{5}{*}{0.95} & $\hat{\beta}_{L S}$ & 392845.00 & 443548.00 & 444798.00 & 792065.00 & 4038.00 & 11303.00 & 29083.00 & 167873.00 \\
\hline & $\hat{\beta}_{R R}$ & 97097.00 & 124982.00 & 123019.00 & 313791.00 & 917.00 & 5086.00 & 16915.00 & 117076.00 \\
\hline & $\hat{\beta}_{I M R}$ & 156817.00 & 186855.00 & 183490.00 & 412848.00 & 1495.00 & 6407.00 & 19801.00 & 127461.00 \\
\hline & $\hat{\beta}_{I R M}$ & 25807.00 & 31123.00 & 36186.00 & 110867.00 & 242.00 & 1862.00 & 8229.00 & 65067.00 \\
\hline & $\hat{\beta}_{R R}^{*}$ & 1150.97 & 1179.11 & 1994.33 & 8665.58 & 40.57 & 149.42 & 635.75 & 3323.51 \\
\hline \multirow{5}{*}{0.99} & $\hat{\beta}_{L S}$ & 3271226.00 & 3741131.00 & 3216915.00 & 7165352.00 & 43951.00 & 95120.00 & 223294.00 & 1713269.00 \\
\hline & $\hat{\beta}_{R R}$ & 735792.00 & 978962.00 & 840373.00 & 2303451.00 & 10190.00 & 42328.00 & 123189.00 & 1177989.00 \\
\hline & $\hat{\beta}_{J M R}$ & 1132082.00 & 1446732.00 & 1262678.00 & 3179624.00 & 17044.00 & 52675.00 & 144926.00 & 1303063.00 \\
\hline & $\hat{\beta}_{J R M}$ & 178739.00 & 238836.00 & 220975.00 & 701483.00 & 2432.00 & 17802.00 & 55416.00 & 649813.00 \\
\hline & $\hat{\beta}_{R R}^{*}$ & 1058.07 & 1119.13 & 2085.34 & 4880.42 & 45.91 & 129.29 & 431.40 & 2756.36 \\
\hline
\end{tabular}




\section{A V DORUGADE}

Table 6. AMSE of LS and various ridge estimators (With two outlier, $p=4$ and $\left.\beta=(5,8,4,1)^{\prime}\right)$

\begin{tabular}{|c|c|c|c|c|c|c|c|c|c|}
\hline \multirow{2}{*}{\multicolumn{2}{|c|}{$\rho$ Estimator }} & \multicolumn{4}{|c|}{$n=25$} & \multicolumn{4}{|c|}{100} \\
\hline & & $\sigma^{2}=1$ & 5 & 10 & 25 & 1 & 5 & 10 & 25 \\
\hline \multirow{5}{*}{0.85} & $\hat{\beta}_{L S}$ & 41261.00 & 41176.10 & 48149.30 & 43909.90 & 772.00 & 1011.90 & 1660.60 & 6055.50 \\
\hline & $\hat{\beta}_{R R}$ & 14889.10 & 13985.10 & 17189.10 & 17160.50 & 435.00 & 598.70 & 1099.70 & 4683.20 \\
\hline & $\hat{\beta}_{J M R}$ & 20563.80 & 20032.50 & 24093.60 & 23708.50 & 497.90 & 698.30 & 1243.60 & 4979.50 \\
\hline & $\hat{\beta}_{J R M}$ & 7908.00 & 7977.00 & 10424.80 & 12221.80 & 341.00 & 457.20 & 823.40 & 3597.20 \\
\hline & $\hat{\beta}_{R R}^{*}$ & 6332.80 & 6256.90 & 9736.60 & 11913.90 & 320.10 & 436.00 & 773.30 & 3101.60 \\
\hline \multirow{5}{*}{0.90} & $\hat{\beta}_{L S}$ & 145383.00 & 152150.00 & 171664.00 & 216624.00 & 3410.00 & 3734.00 & 6045.00 & 20448.00 \\
\hline & $\hat{\beta}_{R R}$ & 33303.10 & 33452.10 & 47627.60 & 67027.20 & 1361.90 & 1626.20 & 3080.40 & 13200.30 \\
\hline & $\hat{\beta}_{J M R}$ & 52887.70 & 58909.10 & 70394.90 & 93015.60 & 1760.80 & 2070.50 & 3715.10 & 14821.50 \\
\hline & $\hat{\beta}_{J R M}$ & 11866.90 & 12645.60 & 17357.20 & 28180.90 & 569.60 & 799.60 & 1567.10 & 7411.50 \\
\hline & $\hat{\beta}_{R R}^{*}$ & 5511.50 & 6470.50 & 8104.90 & 14333.30 & 291.80 & 477.70 & 817.90 & 2695.30 \\
\hline \multirow{5}{*}{0.95} & $\hat{\beta}_{L S}$ & 1980295.00 & 1955972.00 & 1546191.00 & 2190932.00 & 27950.00 & 29444.00 & 45744.00 & 178431.00 \\
\hline & $\hat{\beta}_{R R}$ & 509210.00 & 459140.00 & 326485.00 & 605908.00 & 8348.00 & 9357.00 & 18715.00 & 107883.00 \\
\hline & $\hat{\beta}_{J M R}$ & 792571.00 & 745312.00 & 548092.00 & 918717.00 & 12725.00 & 13522.00 & 24832.00 & 124177.00 \\
\hline & $\hat{\beta}_{J R M}$ & 137224.00 & 105464.00 & 76545.00 & 176526.00 & 2448.00 & 2937.00 & 6655.00 & 50663.00 \\
\hline & $\hat{\beta}_{R R}^{*}$ & 6753.40 & 5063.10 & 7185.50 & 10095.80 & 285.20 & 389.60 & 917.90 & 3859.40 \\
\hline \multirow{5}{*}{0.99} & $\hat{\beta}_{L S}$ & 15458210.00 & 15216186.00 & 15121157.00 & 18711248.00 & 289717.00 & 365075.00 & 439706.00 & 1939908.00 \\
\hline & $\hat{\beta}_{R R}$ & 3941721.00 & 3719049.00 & 3121535.00 & 4240048.00 & 81054.00 & 127113.00 & 181203.00 & 1214587.00 \\
\hline & $\hat{\beta}_{J M R}$ & 6116418.00 & 6002584.00 & 5272954.00 & 6857110.00 & 116202.00 & 181722.00 & 236596.00 & 1377073.00 \\
\hline & $\hat{\beta}_{J R M}$ & 1104532.00 & 1048186.00 & 735478.00 & 1104213.00 & 20517.00 & 38537.00 & 63692.00 & 611190.00 \\
\hline & $\hat{\beta}_{R R}^{*}$ & 5809.60 & 6557.80 & 9078.90 & 15027.20 & 284.50 & 419.00 & 660.70 & 3859.90 \\
\hline
\end{tabular}

From AMSE values reported in Tables 5 and $6, \hat{\beta}_{J R M}$ gives better performance than $\hat{\beta}_{L S}, \hat{\beta}_{R R}$ and $\hat{\beta}_{J M R}$. However, proposed estimator $\hat{\beta}_{R R}^{*}$ gives better performance than $\hat{\beta}_{L S}, \hat{\beta}_{R R}$ and $\hat{\beta}_{J M R}$ including $\hat{\beta}_{J R M}$ for all combinations of $\rho, n, \sigma^{2}$ and $V$. Particularly, $\hat{\beta}_{R R}^{*}$ having the significantly less AMSE values as compare to other estimators for all combinations of $\rho, n, \sigma^{2}$ and $V$. 


\section{IMPROVED RIDGE ESTIMATOR IN LINEAR REGRESSION}

Case V. As with heteroscedastic and/or correlated errors, departure from the normality assumption is also one of the common problems in regression. Assume $\varepsilon$ follows a non-normal distribution. To examine the robustness of all estimators under consideration, random numbers are generated for the error terms $(\varepsilon)$ from each of the $t, F$, Chi-square and exponential distributions respectively. The AMSE was computed for various values of triplet $(\rho, n$, distribution of $\varepsilon$ ) for $p=4$ and reported in Table 7.

Table 7. AMSE of LS and various ridge estimators $\left(p=4\right.$ and $\left.\beta=(6,3,5,3)^{\prime}\right)$

\begin{tabular}{|c|c|c|c|c|c|c|c|c|c|}
\hline \multirow{2}{*}{$\rho$} & \multirow{2}{*}{ Estimator } & \multicolumn{4}{|c|}{$n=25$} & \multicolumn{4}{|c|}{100} \\
\hline & & $\varepsilon \sim \operatorname{Chi}(6)$ & $F(2,3)$ & $\mathrm{T}(8)$ & $\operatorname{Exp}(4)$ & Chi(6) & $F(2,3)$ & $\mathrm{T}(8)$ & $\operatorname{Exp}(4)$ \\
\hline \multirow{5}{*}{0.85} & $\hat{\beta}_{L S}$ & 76.24 & 89.45 & 4.61 & 108.34 & 52.91 & 93.15 & 62.91 & 871.96 \\
\hline & $\hat{\beta}_{R R}$ & 64.27 & 35.53 & 3.59 & 38.46 & 20.91 & 68.10 & 60.16 & 330.95 \\
\hline & $\hat{\beta}_{J M R}$ & 64.32 & 45.86 & 3.37 & 51.85 & 25.30 & 71.55 & 59.56 & 463.09 \\
\hline & $\hat{\beta}_{J R M}$ & 68.43 & 49.71 & 4.05 & 56.37 & 32.26 & 75.46 & 61.46 & 486.09 \\
\hline & $\hat{\beta}_{R R}^{*}$ & 58.16 & 17.56 & 2.58 & 15.95 & 8.35 & 56.71 & 57.78 & 173.11 \\
\hline \multirow{5}{*}{0.90} & $\hat{\beta}_{L S}$ & 189.40 & 494.30 & 30.00 & 607.00 & 254.00 & 290.20 & 97.60 & 4609.90 \\
\hline & $\hat{\beta}_{R R}$ & 99.40 & 159.70 & 14.30 & 157.20 & 72.80 & 124.30 & 71.70 & 1286.50 \\
\hline & $\hat{\beta}_{J M R}$ & 122.50 & 255.90 & 15.90 & 263.70 & 108.90 & 168.50 & 75.10 & 2153.00 \\
\hline & $\hat{\beta}_{J R M}$ & 119.90 & 233.70 & 19.60 & 257.90 & 124.40 & 162.30 & 78.90 & 2053.30 \\
\hline & $\hat{\beta}_{R R}^{*}$ & 59.43 & 16.93 & 4.59 & 13.71 & 10.56 & 57.36 & 59.62 & 170.73 \\
\hline \multirow{5}{*}{0.95} & $\hat{\beta}_{L S}$ & 1597.00 & 4623.00 & 325.00 & 6546.00 & 3147.00 & 2834.00 & 609.00 & 48742.00 \\
\hline & $\hat{\beta}_{R R}$ & 516.00 & 1162.00 & 89.00 & 1678.00 & 722.00 & 876.00 & 224.00 & 12090.00 \\
\hline & $\hat{\beta}_{J M R}$ & 862.00 & 2160.00 & 157.00 & 3054.00 & 1344.00 & 1506.00 & 325.00 & 22501.00 \\
\hline & $\hat{\beta}_{J R M}$ & 737.00 & 1830.00 & 138.00 & 2679.00 & 1223.00 & 1238.00 & 296.00 & 19425.00 \\
\hline & $\hat{\beta}_{R R}^{*}$ & 57.66 & 12.49 & 5.11 & 9.28 & 5.74 & 54.17 & 59.05 & 121.59 \\
\hline \multirow{5}{*}{0.99} & $\hat{\beta}_{L S}$ & 19447.00 & 47712.00 & 4916.00 & 64387.00 & 39836.00 & 27943.00 & 5447.00 & 526282.00 \\
\hline & $\hat{\beta}_{R R}$ & 6080.00 & 13278.00 & 1596.00 & 15450.00 & 11031.00 & 6690.00 & 1575.00 & 136786.00 \\
\hline & $\hat{\beta}_{J M R}$ & 10382.00 & 23410.00 & 2852.00 & 27852.00 & 19415.00 & 12269.00 & 2660.00 & 243775.00 \\
\hline & $\hat{\beta}_{J R M}$ & 8360.00 & 20304.00 & 2286.00 & 23779.00 & 16420.00 & 11426.00 & 2313.00 & 210271.00 \\
\hline & $\hat{\beta}_{R R}^{*}$ & 57.04 & 11.86 & 5.05 & 9.27 & 5.41 & 53.30 & 58.91 & 113.13 \\
\hline
\end{tabular}




\section{A V DORUGADE}

From Table 7, $\hat{\beta}_{R R}^{*}$ gives better performance than $\hat{\beta}_{L S}, \hat{\beta}_{R R}, \hat{\beta}_{J M R}$ and $\hat{\beta}_{J R M}$ for various values of triplet $\left(\rho, n\right.$, distribution of $\varepsilon$ ). This indicates $\hat{\beta}_{R R}^{*}$ is not sensitive to the departure from the normality assumption of error terms.

From Case $\mathrm{I}$ to $\mathrm{V}$ in above simulation study, it is observed that $\hat{\beta}_{L S}$ is sensitive in each above case and produces unreliable results. Among estimators $\hat{\beta}_{R R}, \hat{\beta}_{J M R}$ and $\hat{\beta}_{J R M}$ no any estimator is better in each of the above cases. But $\hat{\beta}_{R R}^{*}$ is superior than other estimators in each of the above cases for different combinations of size of the sample $(n)$, level of multicollinearity $(\rho)$, variance of the error term $\left(\sigma^{2}\right)$, number of predictors $(p)$, matrix $V$ and number of outliers. The novel feature of the proposed estimator is that it can be used without any modification in the proposed estimator it is better alternative to combat one or more than one problems among multicollinearity, outliers, heteroscedastics and/or correlated errors and departure from the normality assumption.

\section{Conclusion}

A new estimation method for the ridge parameter and hence the ridge regression estimator $\hat{\beta}_{R R}^{*}$ was introduced. A simulation study indicated $\hat{\beta}_{R R}^{*}$ gave better performance than other estimators used when the model defined in linear regression exhibits multicollinearity and heteroscedastic and/or correlated errors, non-normal errors, and outliers. The proposed estimator performed well compared with the alternatives considered, and should be useful for practitioners.

\section{References}

Alheety, M. I., \& Kibria, B. M. G. (2009). On the Liu and almost unbiased Liu estimators in the presence of multicollinearity with heteroscedastic or correlated errors. Surveys in Mathematics and its Applications, 4, 155-167.

Alkhamisi, M. A., \& Shukur, G. (2007). Monte Carlo study of recent ridge parameters. Communications in Statistics - Simulation and Computation, 36(3), 535-547. doi: 10.1080/03610910701208619

Al-Hassan, Y. M. (2010). Performance of a new ridge regression estimator. Journal of the Association of Arab Universities for Basic and Applied Sciences, 9(1), 23-26. doi: 10.1016/j.jaubas.2010.12.006 


\section{IMPROVED RIDGE ESTIMATOR IN LINEAR REGRESSION}

Batah, F. S. M., Ramnathan, T. V., \& Gore, S. D. (2008). The efficiency of modified jackknife and ridge type regression estimators: A comparison. Surveys in Mathematics and its Applications, 3, 111-122.

Bayhan, G. M., \& Bayhan, M. (1998). Forecasting using autocorrelated errors and multicollinear predictor variables. Computers \& Industrial Engineering, 34(2), 413-421. doi: 10.1016/S0360-8352(97)00278-7

Birkes, D., \& Dodge, Y. (1993). Alternative Methods of Regression. New York, NY: John Wiley and Sons.

Dorugade, A. V., \& Kashid, D. N. (2010). Alternative method for choosing ridge parameter for regression. Applied Mathematical Sciences, 4(9), 447-456.

Firinguetti, L. (1989). A simulation study of ridge regression estimators with autocorrelated errors. Communications in Statistics - Simulation and Computation, 18(2), 673-702. doi: 10.1080/03610918908812784

Hampel, F. R., Ronchetti, E. M., Rousseeuw, P. J., \& Stahel, W. A. (1986). Robust statistics: The approach based on influence function. New York, NY: John Wiley.

Hoerl, A. E., \& Kennard, R. W. (1970). Ridge regression: Biased estimation for nonorthogonal problems. Technometrics, 12(1), 55-67.

doi: 10.1080/00401706.1970.10488634

Hoerl, A. E., Kennard, R. W., \& Baldwin, K. F. (1975). Ridge regression: Some simulations. Communications in Statistics, 4(2), 105-123.

Huber, P. J. (1981). Robust Statistics. New York, NY: John Wiley.

Jadhav, N. H., \& Kashid, D. N. (2011). A jackknifed ridge m-estimator for regression model with multicollinearity and outliers. Journal of Statistical Theory and Practice, 5(4), 659-673. doi: 10.1080/15598608.2011.10483737

Khalaf, G., \& Shukur, G. (2005). Choosing ridge parameter for regression problems. Communications in Statistics - Theory and Methods, 34(5), 1177-1182. doi: 10.1081/STA-200056836

Kibria, B. M. G. (2003). Performance of some new ridge regression estimators. Communications in Statistics - Simulation and Computation, 32(2), 419-435.doi: 10.1081/SAC-120017499

Lawless, J. F., \& Wang, P. (1976). Simulation study of ridge and other regression estimators. Communications in Statistics - Theory and Methods, 5(4), 307-323. doi: 10.1080/03610927608827353

Li, Y., \& Yang, H. (2011). On the performance of the jackknifed modified ridge estimator in the linear regression model with correlated or heteroscedastic 


\section{A V DORUGADE}

errors. Communications in Statistics - Theory and Methods, 40(15), 2695-2708. doi: 10.1080/03610926.2010.491589

McDonald, G. C., \& Galarneau, D. I. (1975). A Monte Carlo evaluation of some ridge-type estimators. Journal of the American Statistical Association, 70(350), 407-416. doi: 10.1080/01621459.1975.10479882

Montgomery, D. C., Peck, E. A., \& Vining, G. G. (2001). Introduction to Linear Regression Analysis ( $3^{\text {rd }}$ ed.). New York, NY: John Wiley.

Muniz, G., \& Kibria, B. M. G. (2009). On some ridge regression estimators: An empirical comparisons. Communications in Statistics - Simulation and Computation, 38(3), 621- 630. doi: 10.1080/03610910802592838

Muniz, G., Kibria, B. M. G., Mansson, K., \& Shukur, G. (2012). On developing ridge regression parameters: A graphical investigation. SORT: Statistics and Operations Research, 36(2), 115-138.

Özkale, M. R. (2008). A jackknifed ridge estimator in the linear regression model with heteroscedastic or correlated errors. Statistics \& Probability Letters, 78(18), 3159-3169. doi: 10.1016/j.spl.2008.05.039

Rousseeuw, P. J., \& Leroy, A. M. (1987). Robust regression and outlier detection. New York, NY: John Wiley and Sons.

Singh, B., \& Chaubey, Y. P. (1987). On some improved ridge estimators. Statistische Hefte, 28(1), 53-67. doi: 10.1007/bf02932590.

Trenkler, G. (1984). On the performance of biased estimators in the linear regression mode with correlated or heteroscedastic errors. Journal of Econometrics, 25(1/2), 179-190. doi: 10.1016/0304-4076(84)90045-9

Yang, H., \& Chang, X. (2010). A new two-parameter estimator in linear regression. Communications in Statistics - Theory and Methods, 39(6), 923-934. doi: 10.1080/03610920902807911 